\title{
(息)
}

Citation:

Bardehle, D and Dinges, M and White, A (2015) Was ist Männergesundheit? Eine Definition [What is Men's Health? A definition]. Gesundheitswesen (Bundesverband der Arzte des Offentlichen Gesundheitsdienstes (Germany)), 78 (07). e30-e39. ISSN 0941-3790 DOI: https://doi.org/10.1055/s0035-1564077

Link to Leeds Beckett Repository record:

https://eprints.leedsbeckett.ac.uk/id/eprint/1352/

Document Version:

Article (Accepted Version)

The aim of the Leeds Beckett Repository is to provide open access to our research, as required by funder policies and permitted by publishers and copyright law.

The Leeds Beckett repository holds a wide range of publications, each of which has been checked for copyright and the relevant embargo period has been applied by the Research Services team.

We operate on a standard take-down policy. If you are the author or publisher of an output and you would like it removed from the repository, please contact us and we will investigate on a case-by-case basis.

Each thesis in the repository has been cleared where necessary by the author for third party copyright. If you would like a thesis to be removed from the repository or believe there is an issue with copyright, please contact us on openaccess@leedsbeckett.ac.uk and we will investigate on a case-by-case basis. 


\section{Was ist Männergesundheit? Eine Definition}

\section{Zusammenfassung}

\section{Ziel der Studie}

Männergesundheit gerät immer stärker in den Fokus von Gesundheitsforschung, Epidemiologie, Gesundheitspflegeorganisation und Gesundheitspolitik. In den vergangenen 10-15 Jahren wurden von einigen Ländern und der EU Männergesundheitsberichte publiziert, in denen auf Probleme der Gesundheit beim Mann hingewiesen wurden. Ausgangspunkt für eine wissenschaftliche Untersuchung von Männergesundheit ist die Definition des Begriffs „Männergesundheit". Eine Gruppe von deutschen Experten auf dem Gebiet von Männergesundheit hat sich dieser Aufgabe angenommen.

\section{Methodik}

Eine Literaturrecherche in internationalen und nationalen Datenbanken erfolgte für die Jahre 1990 bis 2014 nach ausgewählten MeSH Terms und nach „Experten für Männergesundheit“, nach "grauer Literatur", in Gesundheitsberichten und Webseiten.

Es folgte eine Expertenkonferenz zur Definitionsfindung mittels modifizierter Delphi Methode nach W. Zinn. Von der Expertengruppe wurden Mindestkriterien für eine Definitions Männergesundheit erstellt und diskutiert, denen die neue Definition von Männergesundheit genügen muss. Daraus wurde in der dritten Runde schrittweise eine Definition erstellt.

\section{Ergebnisse}

Die internationale Literaturrecherche ergab sieben Definitionen zu "Men's Health" in der englischsprachigen und fünf Definitionen in der deutschen inkl.in der „grauen“ Literatur. Auf Grund der von der Expertengruppe erarbeiteten 7 Mindestkriterien wurden 15 relevante Themengebiete gelistet, von denen durch Gewichtung acht Themengebiete als relevant für eine neue Definition angesehen wurden. Im Abgleich mit den bisherigen Definitionen konnte keine diese acht Kriterien erfüllen. Deshalb wurde im nächsten Arbeitsschritt von der Expertengruppe eine neue Definition Männergesundheit ausgearbeitet. Die Definition hat Bezug zum WHO-Begriff "Gesundheit" und beinhaltet Dimensionen von Gesundheit und Krankheit, Risiko- und Schutzfaktoren, die spezielle Präventions- und Versorgungsangebote über alle Lebensphasen erfordern. Alle Teilnehmer der Expertengruppe stimmten der erarbeiteten Definition zu.

\section{Schlussfolgerung}

Die Definition von Männergesundheit stellt eine Basis für weitere wissenschaftliche Arbeiten und die Praxis dar, um Männergesundheit in Deutschland und weiteren Ländern zu verbessern.

\section{Schlüsselwörter}

Männergesundheit, Gesundheitsressourcen, Lebensstil, Lebensqualität, Gesundheitsangebote für Männer 


\section{(Englische Fassung des Abstracts)}

What is Men's Health? A definition

\section{Summary}

Target of the study

Men's Health is increasingly coming into focus of health research, epidemiology, health care organization, and health policy. Over the past 10-15 years have been published by some 
countries and the EU Men's Health reports where problems of health noted in men. Starting point for a scientific study of men's health is the definition of "Men's Health". A group of German experts in the field of men's health has taken on this task.

\section{Methodology.}

A literature review in international and national databases was performed for the years 1990 to 2014 by selected MeSH terms and for "experts for men's health" . Another research concerned "gray literature" in Germany, especially health reports and web pages. This was followed by a conference of experts to define "Men's Health" using a modified Delphi method according to W. Zinn.

From the expert group minimum criteria for a definition "Men's Health" were created and discussed, which must comply with the new definition of men's health. This definition was created in the third round of the Delphi method.

\section{Results}

The international literature review yielded seven definitions of "Men's Health" in the Englishspeaking and five definitions in the German inkl.in the "gray" literature. Due to the developed by the Expert Group 7 minimum criteria 15 relevant topics were listed, of which by weighting 8 subject areas were considered relevant for a new definition. In comparison with the previous definitions none could fulfill these eight criteria. Therefore, in the next step of the expert group a new definition "Men's Health" was elaborated. The definition has reference to the WHO concept of "health" and includes dimensions of health and disease, risk and protective factors that require special prevention and care services for all phases of life. All participants in the expert group agreed on the definition developed.

\section{Conclusions}

The definition of Men's Health is a basis for further scientific work and practice to improve men's health in Germany and other countries.

Keywords

Men's Health, Health resources, Lifestyle, Quality of life, Delivery of health services for men 


\section{Mini Abstract mit max 500 Zeichen in Deutsch für ELPS Publikation}

Was ist Männergesundheit? Eine Definition

\section{Autoren}

E-Mail: bardehle@stiftung-maennergesundheit.de; Doris Bardehle, apl. Prof.

Dr.med., Koordinatorin des Wissenschaftlichen Beirates der Stiftung

Männergesundheit, Berlin

E-Mail: martin.dinges@igm-bosch.de; Martin Dinges, Prof. Dr.phil., Stellvertretender Leiter des Institutes für Geschichte der Medizin der Robert-Bosch-Stiftung, Stuttgart und apl. Professor für Neuere Geschichte an der Universität Mannheim

E-Mail: A.White@leedsbeckett.ac.uk; Alan White, Professor, Centre for Men's health, Institute for Health and Wellbeing, Faculty of Health and Social Sciences, Leeds Beckett University, Leeds, United Kingdom 
Korrespondenzadresse

Prof. Dr. Doris Bardehle

Stiftung Männergesundheit

Claire-Waldoff-Str. 3

10117 Berlin, Germany

Tel.: ++49 (30) 652 126-120

Fax: ++49 (30) 652 126-112

bardehle@stiftung-maennergesundheit.de

Eine Expertengruppe analysierte 7 englischsprachige und 5 deutsche Definitionen von Männergesundheit. Mit einem modifizierten Delphi-Verfahren wurde eine Rangliste von 8 Kriterien für einen Definitionsvorschlag auf der Basis des Salutogenesemodells, des WHOBegriffs von Gesundheit und von Dimensionen von Gesundheit und Krankheit, die insbesondere für Männer und Jungen relevant sind erarbeitet. Die Definition sollte diskutiert und durch Aktionsprogramme für Männer umgesetzt werden.

Schlüsselwörter: Männergesundheit, Gesundheitsressourcen, Lebensstil, Lebensqualität, Gesundheitsangebote für Männer

\section{Mini Abstract mit max 500 Zeichen in Englisch für ELPS Publikation}

\section{What is Men's Health? A definition}

\section{Authors}

E-Mail: bardehle@stiftung-maennergesundheit.de; Doris Bardehle, apl. Prof.

Dr.med., Co-ordinator of the Scientific Board of the Foundation of Men's Health, Berlin

E-Mail: martin.dinges@igm-bosch.de; Martin Dinges, Prof. Dr.phil., Deputy Head of the Institute for the History of Medicine, Robert-Bosch-Foundation, Stuttgart

E-Mail: A.White@leedsbeckett.ac.uk; Alan White, Professor, Centre for Men's health, Institute for Health and Wellbeing, Faculty of Health and Social Sciences, Leeds Beckett University, Leeds, United Kingdom

Address for correspondence

Prof. Dr. Doris Bardehle

Stiftung Männergesundheit

Claire-Waldoff-Str. 3 
10117 Berlin, Germany

Tel.: ++49 (30) 652 126-120

Fax: ++49 (30) 652 126-112

bardehle@stiftung-maennergesundheit.de

An expert group analyzed 7 English-language and 5 German definitions of men's health. With a modified Delphi method a ranking list with 8 criteria for a new proposed definition for Men's Health was elaborated. The platform of the new definition are the model of salutogenesis, the WHO-definition of health and the dimensions of health and disease, which are particularly relevant for men and boys. The definition should be discussed and put into practice via health action plans for men.

Key words: Men's Health, Health resources, Lifestyle, Quality of life, Delivery of health services for men

\section{Was ist Männergesundheit? Eine Definition}

\section{Einleitung}

Im Jahr 2004 schrieb Alan White im Editorial des neu gegründeten Journals of Men's Health and Gender, dass Männergesundheit ein wachsendes Problem darstellt und auf akademisches und klinisches Interesse gestoßen sei [1]. Die Entwicklung einer Definition von Männergesundheit habe erst in den letzten 10-20 Jahren begonnen. Es wurde bald klar, dass eine Definition mehr enthalten müsse als männerspezifische Erkrankungen [1].

Die erste bekannte Definition Männergesundheit stammt von R. Fletcher aus Australien aus dem Jahr 1997 und bezog sich überwiegend auf männerspezifische Erkrankungen, Risikofaktoren und Interventionen [2].

Einen wichtigen Schritt vorwärts bedeutet die Definition Männergesundheit des Men's Health Forum aus England aus dem Jahr 2004, die in dem Dokument „Getting it sorted: a policy programme for men's health“ verankert ist und physische, psychologische, soziale und Umweltfaktoren in die Definition Männergesundheit einschloss [3].

Dennoch schrieb im Jahr 2007 D. J. Porche im Editorial des neu gegründeten American Journal of Men's Health, dass es keine eindeutige Definition von „Männergesundheit" gibt. („There are no clear and consistent definitions of what constitutes men's health”). Das war 
die Schlussfolgerung einer internationalen Übersicht von Definitionen von

Männergesundheit [4].

Der Vorschlag von D. J. Porche beinhaltet einen ganzheitlichen und umfassenden Ansatz, der psychische, mentale, emotionale, soziale und spirituelle Lebenserfahrungen sowie die Bedürfnisse von Männern in allen Lebensabschnitten einschließt [5].

Warum bemühen sich gegenwärtig mehrere Länder, Männergesundheit zu identifizieren? James A Smith schrieb bereits im Jahr 2007, dass das Fehlen einer allgemein akzeptierten Definition von Männergesundheit problematisch für die Etablierung einer staatlichen und föderalen Männergesundheitspolitik ist. Es geht auch um solche Fragen, wie Männer ihre Gesundheit sehen und wie der Bezug zur Inanspruchnahme von Gesundheitsleistungen aus Public Health Perspektive zu sehen ist [6].

Inzwischen gibt es für Australien und für Irland männerbezogene gesundheitspolitische Programme [7].

In dieser Publikation gibt es einen Hinweis aus dem irischen Strategiedokument zur Männergesundheit, dass eine Strategie nicht von einer ständigen Rechtfertigung von männlichen Verhaltensweisen zu ihrer Gesundheit auszugehen hat (S. 425) (it is against particular norms of masculine behaviour that men must constantly negotiate their own behaviour in relation to how they manage their health).

In den vergangenen Jahren wurden Männergesundheitsberichte für Deutschland [8; 9; 10], für Europa [11], für Asien [12] und für andere europäische Länder publiziert, demnächst für die USA. Einige dieser Berichte enthalten eine Definition Männergesundheit.

In den vergangenen zwei Jahren wurde Männergesundheit in den USA durch mehrere Aktivitäten etabliert: Es wurde die „Foundation of Men's Health (USA)“ gegründet, die Leitung der "International Society of Men's Health (ISMH)“ von Österreich übernommen, der „American Urological Association (AUA)“ wurde die Leitfunktion für Männergesundheit übertragen und es wurde die „American Society of Men's Health“ gegründet. Die „International Consultation Company on Urological Diseases in Men's Health and Infertility Men's Health Initiative" (ICUD-MHI)“ wird den Prozess zur Männergesundheit in den USA koordinieren, der bereits zu einer einvernehmlichen Neudefinition von Männergesundheit für die USA geführt hat [13].

Definitionen zur Männergesundheit in Deutschland wurden von der Stiftung Männergesundheit initiiert. Entsprechend der internationalen Entwicklung von Definitionen zur Männergesundheit bestand der Bedarf, die im Jahre 2006 bei der Gründung der Stiftung Männergesundheit erarbeitete Definition durch eine aktuellere zu ersetzen, die der internationalen Entwicklung angepasst ist und für gesundheitspolitische Strategiebildungen geeignet ist.

Männergesundheit rückt auch in Deutschland schrittweise in den Fokus der Gesundheitspoltik. Bei der Analyse der geschlechtsspezifischen gesundheitlichen Lage der Bevölkerung wird gegenwärtig ein Unterschied in der Lebenserwartung von 5 Jahren zum 
Nachteil der Männer festgestellt. Die Analyse der gesundheitlichen Lage von Männern und Frauen zeigt einerseits vergleichbare, andererseits aber unterschiedliche Muster in der Morbiditätsstruktur, im Verhalten, bei Risikofaktoren und für sozio-demographische Gruppen. Unterschiede gibt es im Inanspruchnahmeverhalten ambulanter ärztlicher Leistungen und bei Präventions- und Vorsorgemaßnahmen.

Die Definition von Gesundheit der WHO aus dem Jahr 1946 [14] „Die Gesundheit ist ein Zustand des vollständigen körperlichen, geistigen und sozialen Wohlergehens und nicht nur das Fehlen von Krankheit oder Gebrechen" dient als Grundlage für eine Definition von Männergesundheit. Die WHO-Definition ist eine grundsätzliche Feststellung, aber sie wurde nicht geschlechtsspezifisch formuliert. Unser aktuelles Wissen über Unterschiede im Gesundheitsstatus von Männern und Frauen erfordert eine geschlechtsspezifische Sicht und auch spezifische Definitionen von „Männergesundheit" und „Frauengesundheit".

Das Ziel der Publikation besteht darin, anhand einer Analyse der vorliegenden internationalen und nationalen Definitionen zu Männergesundheit eine Anpassung und Weiterentwicklung der in Deutschland vorhandenen Definitionen von Männergesundheit an den wissenschaftlichen Erkenntnisstand vorzunehmen und zur Diskussion zu stellen. Die Einbeziehung der Gesundheitsversorgung als Bestandteil der Definition von Männergesundheit wird in der Literatur unterschiedlich gesehen. Folgende Fragen sollten versucht werden zu beantworten:

a) Welchen Kriterien sollte eine Definition von Männergesundheit genügen?

b) Gehört die Anpassung der Gesundheitsversorgung an männliche Verhaltensmuster zur Definition von Gesundheitsversorgung?

\section{Methodik}

Der Arbeit liegen umfassende Literaturrecherchen nach Definitionen „Männergesundheit" in internationalen und nationalen Datenbanken, in "grauer Literatur" in deutscher Sprache zu Grunde sowie eine Expertenberatung zur Definitionsfindung nach einem DelphiVerfahren. Die vier methodischen Grundlagen werden getrennt ausgewertet.

\section{Internationale Literaturrecherche}

Die Internationale Literaturrecherche erfolgte durch eine PubMed Recherche (englisch)für die jahre 1990 - 2014, für die geeignete MeSH Terms verwendet wurden.

\section{Fußnote ( ${ }^{*}$ Vorschlag von MeSH terms vom Senior MeSH Analyst Dan Cho, Ph.D. der National Library, National Institutes of Health)}

Folgende MeSH Terms wurden für die PubMed-Recherche der Jahre 1990 bis 2014 genutzt: 1. Men's health AND 1990-2014 
2. Gender Identity AND 1990-2014

3. Men's health AND gender AND 1990-2014

Zusätzlich wurde nach folgenden Begriffen gefragt, um die Trefferzahl zu reduzieren

- defini* AND gender identity AND men's health AND 1990-2014

- men's health AND gender identity AND 1990-2014

- "men's health defini*" AND gender identity AND 1990-2014

Weitere Abfragen bezogen sich auf eine internationale Autorenrecherche, die folgende ausgewiesene Experten für Männergesundheit einschloss:

- Prof. Dr. Demetrius J Porche: Begründer des American Journals of Men's Health 2007, und der Chief Editor dieser Zeitschrift.

- Dr. Richard Fletcher: Fathers and Families Research Program. Family Action Centre, Faculty of Health, the University of Newcastle; Australia

- James A Smith: Department of Paramedic \& Social Health Sciences, Flinders University, Adelaide, Australia

- Dr. Noel Richardson: Director of the Centre for Men's Health at the Institute of Technology Carlow and the editor of the first National Policy on Men's Health, 2008-2013, Ireland

- Prof. Dr. Alan White: Director of the Centre for Men's Health. Institute for Health and Wellbeing, Faculty of Health and Social Sciences. Leeds Beckett University, UK. Chairman of the Global Committee "Unmet Needs in Men's Health". Main editor of the "European State of Men's Health Report 2011", UK

- Prof. Dr. Ridwan Shabsigh: Dept. of surgery at St. Barnabas Hospital, Prof. of urology at Cornell University New York. Editor of Journal of Men's Health, US. Member of the Editorial Committee "Unmet Needs in Men's Health", The President of the International Society of Men's Health, USA

- Prof. Dr. Tan Hui Meng: Medical and Education Development Department, Faculty of Medicine, University of Malaya, Kuala Lumpur, Malaysia and Subang Jaya Medical Centre, Subang Jaya, Malaysia, Main editor of the Asian Men's Health Report 2013, Malaysian Men's Health Initiative of the Malaysian Society of Andrology and the Study of the Aging Male (MSASAM). University of Malaya

- Prof. Dr. Larry Goldenberg, British Columbia, Head of the Department of Urologic Sciences, University of British Columbia, Vancouver; Member of the Committee "Unmet Needs in Men's Health", Canada

Weitere Recherchen bezogen sich auf die WHO, das Men's Health Forum in UK (menshealthforum.org.uk), das European Mens Health Forum (emhf.org), die Webseite der International Society of Men's Health (WWW.ismh.org) und die Webseite der American 
Society of Men's Health (WWW.asmhnet.org).

\section{Nationale Literaturrecherche}

Für die deutschsprachige Literaturrecherche wurden Treffer zu: "Definition Männergesundheit" in Google, Google Scholar, auf der Webseite des Bundesgesundheitsministeriums, bei der BZgA, bei Wikipedia und in den Datenbanken des DIMDI gesucht.

\section{Expertenkonferenz zur Definitionsfindung mittels modifizierter Delphi Methode}

Zur Formulierung der Definition wurde eine nach Prof. Dr. Zinn modifizierte Delphi Methode eingesetzt. Während die klassische Delphi Methode ausschließlich auf schriftlichem wege durchgeführt wird, wie Bortz D and Döring erläutern [15], erfolgte hier die Abstimmung in einer kombinierten Form der individuellen schriftlichen Bearbeitung der Fragestellung und anschließender Gruppendiskussion.

Als Experten standen Autoren des Ersten und Zweiten Männergesundheitsberichtes der Stiftung Männergesundheit und Mitglieder des wissenschaftlichen Beirates der Stiftung Männergesundheit zur Verfügung. Die Begleitung der Gruppe durch das modifizierte Delphi Verfahren erfolgte durch Prof. Zinn (Tab. 3 Auflistung der Experten).

In der ersten Runde legte jeder Experte schriftlich fest, was für ihn die Mindestkriterien sind, denen die neu zu entwickelnde Definition genügen muss.

Die Mindestkriterien wurden anschließend visualisiert und der Gruppe zur Diskussion zur Verfügung gestellt, um dann ausführlich erörtert zu werden. Die Experten einigten sich auf sieben Mindestkriterien, denen eine neue Definition von Männergesundheit genügen muss. Die kritische Durchsicht bestehender Definitionen ergab, dass keine den sieben Kriterien genügte.

In der zweiten Runde fertigte jeder Experte schriftlich eine individuelle Themensammlung an, die zur Definition gehören. Nun hatten die Experten wieder die Möglichkeit, die Inhalte in der Gruppe zu erläutern. Überschneidungen wurden herausgefiltert und Unklarheiten präzisiert. Es folgte dann eine Gewichtung der Themen durch die Experten, um irrelevante Inhalte herauszufiltern. Jeder Teilnehmer hatte fünf Punkte zu vergeben, maximal konnten zwei Punkte pro Themengebiet kumuliert werden. Themen, die vier und mehr Punkte erhielten, wurden als Einschlusskriterien für die Definition ausgewählt; Kriterien, die drei oder weniger Punkte erhielten, wurden von der Integration in die Definition ausgeschlossen. 
Tab. 1: Ergebnis der internationalen Literaturrecherche zu $3 \mathrm{MeSH}$ Terms zu "Definition of Men's health" (Nov. 2014)

\begin{tabular}{|c|c|c|c|c|c|}
\hline $\begin{array}{l}\text { Datenbank } \\
\text { / } \\
\text { Quelle }\end{array}$ & $\begin{array}{l}\text { Suchbegri } \\
\text { ff }\end{array}$ & Treffer & $\begin{array}{l}\text { Einschränkun } \\
\text { g des } \\
\text { Begriffs }\end{array}$ & $\begin{array}{l}\text { Tref- } \\
\text { fer }\end{array}$ & Bewertung \\
\hline \multirow[t]{2}{*}{ WHO } & $\begin{array}{l}\text { men's } \\
\text { health }\end{array}$ & 1.010 & $\begin{array}{l}\text { definition } \\
\text { men's health }\end{array}$ & 845 & $\begin{array}{l}\text { Keine Definition bei } \\
\text { fün ausgewählten } \\
\text { Publikationen }\end{array}$ \\
\hline & $\begin{array}{l}\text { male } \\
\text { health }\end{array}$ & 1.040 & $\begin{array}{l}\text { definition } \\
\text { and "male } \\
\text { health" }\end{array}$ & 19 & unpassend \\
\hline \multirow[t]{2}{*}{$\begin{array}{l}\text { European } \\
\text { Union }\end{array}$} & $\begin{array}{l}\text { men's } \\
\text { health }\end{array}$ & 1.139 & $\begin{array}{l}\text { Nicht } \\
\text { möglich }\end{array}$ & & \\
\hline & $\begin{array}{l}\text { Männerges } \\
\text { und-heit }\end{array}$ & $\odot$ & & & \\
\hline Google & $\begin{array}{l}\text { "european } \\
\text { union" } \\
\text { and } \\
\text { definitio } \\
\text { n and } \\
\text { "men's } \\
\text { health" }\end{array}$ & 1.280 .000 & $\begin{array}{l}\text { Durchsuchen } \\
\text { der } \\
\text { erstgelistet } \\
\text { en } \\
\text { Publikation }\end{array}$ & & $\begin{array}{l}1 \text { Definition } \\
\text { (The State of Men's } \\
\text { Health Report, 2011) }\end{array}$ \\
\hline $\begin{array}{l}\text { Men's } \\
\text { Health } \\
\text { Forum }\end{array}$ & $\begin{array}{l}\text { men's } \\
\text { health } \\
\text { definitio } \\
\text { n }\end{array}$ & 4 & & & $\begin{array}{l}1 \text { Definition } \\
\text { (Getting it sorted - } \\
\text { a } \\
\text { policy paper for } \\
\text { men's } \\
\text { health, 2004) }\end{array}$ \\
\hline $\begin{array}{l}\text { European } \\
\text { Men's } \\
\text { health } \\
\text { forum }\end{array}$ & $\begin{array}{l}\text { Keine } \\
\text { Suchmaske }\end{array}$ & - & $\begin{array}{l}\text { Durchsuchen } \\
\text { von drei } \\
\text { relevanten } \\
\text { Publikatione } \\
\text { n }\end{array}$ & & $\begin{array}{l}1 \text { Definition } \\
\text { (Men's Health around } \\
\text { the world, 2009) }\end{array}$ \\
\hline $\begin{array}{l}\text { International } \\
\text { Society of } \\
\text { Men's Healt }\end{array}$ & $\begin{array}{l}\text { definitio } \\
\mathrm{n} \text { men's } \\
\text { health }\end{array}$ & 0 & & & unpassend \\
\hline $\begin{array}{l}\text { American } \\
\text { society of } \\
\text { Men's health }\end{array}$ & $\begin{array}{l}\text { definitio } \\
\mathrm{n} \text { men's } \\
\text { health }\end{array}$ & 0 & & & unpassend \\
\hline \multirow[t]{2}{*}{ PubMed } & $\begin{array}{l}\text { "gender } \\
\text { identity" } \\
\text { AND 1990- } \\
2014\end{array}$ & 257 & $\begin{array}{l}\text { "men`s } \\
\text { health }\end{array}$ & 8 & unpassend \\
\hline & $\begin{array}{l}\text { "men`s } \\
\text { health" } \\
\text { AND 1990- } \\
2014\end{array}$ & 2.128 & $\begin{array}{l}\text { "men`s } \\
\text { health" AND } \\
\text { definition }\end{array}$ & 22 & unpassend \\
\hline
\end{tabular}




\begin{tabular}{|c|c|c|c|c|c|}
\hline & $\begin{array}{l}\text { "male } \\
\text { health" } \\
\text { AND 1990- } \\
2014\end{array}$ & 388 & \begin{tabular}{|l} 
"male \\
health" AND \\
definition
\end{tabular} & 2 & unpassend \\
\hline $\begin{array}{l}\text { Men's and } \\
\text { Boys } \\
\text { Project } \\
\text { Australia }\end{array}$ & & & & & $\begin{array}{l}1 \text { Definition } \\
\text { (Report on men's } \\
\text { health services, } \\
2001 \text { ) }\end{array}$ \\
\hline $\begin{array}{l}\text { Malaysian } \\
\text { Men's } \\
\text { Health } \\
\text { Initiativ } \\
\text { e }\end{array}$ & & & & & $\begin{array}{l}1 \text { Definition } \\
\text { (Asian Men's Health } \\
\text { Report, 2013) }\end{array}$ \\
\hline
\end{tabular}


In der dritten Runde wurde in einer offenen Gruppendiskussion schrittweise eine gemeinsame Formulierung der Definition unter Berücksichtigung der in den ersten Runden vereinbarten Mindestkriterien entwickelt. Die Formulierung erfolgte in einem interaktiven Gruppenprozess, bei dem die Formulierungsvorschläge kontinuierlich für alle sichtbar eingearbeitet und überarbeitet wurden.

In der letzten, ausschließlich schriftlichen Runde wurde der erarbeitete Definitionstext den Experten zur Korrektur übergeben und einvernehmlich von der Expertengruppe akzeptiert.

Die endgültige Fassung der Definition wurde in deutscher und englischer sprache auf der Webseite der stiftung Männergesundheit publiziert.

\section{Ergebnisse \\ Ergebnisse der internationalen Literaturrecherche}

Die internationale (englischsprachige) Literaturrecherche ergab 5 Treffer für die PubMed Recherche und die Recherche in anderen Datenbanken und Quellen

( Tab. 1)

\section{Ergebnisse der nationalen Literaturrecherche}

Die deutschsprachige Literaturrecherche ergab einen Treffer bei Google und zwei Treffer bei Wikipedia. Die Fachliteraturrecherche ergab keine Treffer (Tab. 2).

\section{Internationale Definitionen von Männergesundheit als Ergebnis der Literaturrecherche}

Eine der ersten Definitionen von Männergesundheit kommt aus Australien, New South Wales State, von der Universität Newcastle aus dem Jahr 1997 [2]. Richard Fletcher war in das Projekt "Mens and Boys Project" des Familiy Action Centre der Universität Newcastle involviert und versuchte, Männergesundheit breiter zu fassen als Männerkrankheiten, die sich ausschließlich auf die männliche Anatomie bezogen [2].

\section{Definition Männergesundheit (Australien)}

Richard Fletcher verwendete folgende Definition und publizierte sie im Jahre 1997 als Arbeitsdefinition für Männergesundheit [2]:

„Männergesundheit beinhaltet Krankheiten oder Bedingungen, die nur Männer betreffen, häufiger bei Männern auftreten, ernsthafter bei Männern verlaufen, mit unterschiedlichen Risikofaktoren für Männer oder für die spezifische Interventionen erforderlich sind“ (1. Definition Männergesundheit). 
Tabelle 2: Übersicht über die Literaturrecherche zu deutschsprachiger Literatur

\begin{tabular}{|c|c|c|c|c|c|}
\hline $\begin{array}{l}\text { Datenbank } \\
\text { Quelle }\end{array}$ & Suchbegriff & $\begin{array}{c}\text { Treffer- } \\
\text { zahl }\end{array}$ & $\begin{array}{c}\text { Erweiterung/Einsc } \\
\text { hrän-kung des } \\
\text { Begriffs }\end{array}$ & $\begin{array}{c}\text { Treffer- } \\
\text { zahl }\end{array}$ & Bewertung /Ergebnis \\
\hline Google & $\begin{array}{l}\text { Definition } \\
\text { Männergesund } \\
\text { heit }\end{array}$ & 51.000 & $\begin{array}{l}\text { „Definition } \\
\text { Männergesundheit } \\
\text { “ }\end{array}$ & 10 & 1 Treffer \\
\hline Wikipedia & $\begin{array}{l}\text { Definition } \\
\text { Männergesund } \\
\text { heit }\end{array}$ & 5 & nein & & 2 Treffer \\
\hline $\begin{array}{l}\text { DIMDI } \\
\text { Datenbanke } \\
n\end{array}$ & $\begin{array}{l}\text { Männergesund } \\
\text { heit }\end{array}$ & 1.075 & $\begin{array}{l}\text { Definition } \\
\text { Männergesundheit }\end{array}$ & 17 & 0 Treffer \\
\hline \multirow[t]{2}{*}{$\begin{array}{l}\text { Google } \\
\text { scholar }\end{array}$} & $\begin{array}{l}\text { Männergesund } \\
\text { heit }\end{array}$ & 981 & $\begin{array}{l}\text { Definition } \\
\text { Männergesundheit }\end{array}$ & 375 & \\
\hline & & & $\begin{array}{l}\text { „Definition } \\
\text { Männergesundheit } \\
\text { “ }\end{array}$ & 1 & 0 Treffer \\
\hline $\begin{array}{l}\text { Bundesgesun } \\
\text { dheitsminist } \\
\text { erium }\end{array}$ & $\begin{array}{l}\text { Männergesund } \\
\text { heit }\end{array}$ & 53 & $\begin{array}{l}\text { Definition } \\
\text { Männergesundheit }\end{array}$ & 4 & 0 Treffer \\
\hline BZgA & $\begin{array}{l}\text { Männergesund } \\
\text { heit }\end{array}$ & 21 & $\begin{array}{l}\text { Definition } \\
\text { Männergesundheit }\end{array}$ & 21 & 0 Treffer \\
\hline $\begin{array}{l}\text { Präventologi } \\
\mathrm{n} \\
\text { Gesundheits- } \\
\text { Coach G. J. } \\
\text { Wiggershaus } \\
\text {-Skriboleit }\end{array}$ & $\begin{array}{l}\text { Männergesund } \\
\text { heit }\end{array}$ & 0 & $\begin{array}{l}\text { Definition } \\
\text { Männergesundheit }\end{array}$ & 0 & 0 Treffer \\
\hline
\end{tabular}


Der Hintergrund der ersten Definition von Männergesundheit war die USA Definition von Frauengesundheit, die im Rahmen des Aktionsplans für Frauengesundheit entstand (1991). Die Originaldefinition von Frauengesundheit lautete:

"A women's health issue is a disease or condition unique to women, more prevalent in women, more serious among women, for which risk factors are different for women for which interventions are different for women."

Die deutsche Übersetzung könnte so lauten:

„Frauengesundheit beinhaltet Krankheiten oder Bedingungen, die nur Frauen betreffen, häufiger bei Frauen auftreten, ernsthafter bei Frauen verlaufen, mit unterschiedlichen Risikofaktoren für Frauen oder für die spezifische Interventionen erforderlich sind [2]."

R. Fletcher ergänzte die Definition Männergesundheit um die Begriffe gesundheitliche Lage und gesundheitliche Versorgung, weil er einen Zusammenhang sah. Deshalb definierte er zusätzlich den Begriff:

"Men's Health Service" auf deutsch: Gesundheitsversorgung für Männer mit den drei Schwerpunkten [4]:

i. Ansprechen von Männergesundheitsproblemen

ii. Besondere Beachtung der Männer als Zielgruppe, Einbeziehung der Männer

iii. Die gegenwärtige Versorgung benötigt trotz ihrer Verdienste ein neues Herangehen an Männergesundheit, um den Gesundheitszustand der männlichen Bevölkerung zu verbessern.

\section{Definition Männergesundheit (UK)}

Ähnlich beschrieb das Men's Health Forum of England (2004) Männergesundheit [3; 1]: „Männergesundheit beinhaltet physiologische, psychologische, soziale, kulturelle oder Umweltfaktoren, die einen spezifischen Einfluss auf Jungen oder Männer haben und/oder für die Interventionen erforderlich sind, um eine Verbesserung in der Gesundheit und im Wohlbefinden auf dem individuellen oder Bevölkerungsniveau zu erzielen“ (2. Definition Männergesundheit).

Im Originaltext heißt es:

„A male health issue is one arising from physiological, psychological, social, cultural or environmental factors that have a specific impact on boys or men and / or where particular interventions are required for boys of men in ordert o achieve improvements in health and well-being at either the individual or the population level."

\section{Definition Männergesundheit (USA)}

Porche DJ und Willis DG (2004) fügten zu der letzten Definition den Kontext der Lebensbedingungen hinzu: den Einfluss der Familie, des Wohnumfeldes, der Gesellschaft, 
von Institutionen und der Umwelt [5]. Porche und Willis definierten Männergesundheit folgendermaßen:

„Männergesundheit ist ein ganzheitlicher Prozess, der physische, psychische, emotionale, soziale und spirituelle Lebenserfahrungen und Bedürfnisse über alle Lebensetappen des Mannes betrifft" (3. Definition Männergesundheit).

Im Originaltext heisst es: "Men's Health is a holistic and comprehensive approach that addresses the physical, mental, emotional, social and spiritual life experiences and needs of men throughout their life spans" [5].

\section{Definition Männergesundheit (Irland)}

Irland publizierte europaweit das erste Programm einer Nationalen MännerGesundheitspolitik für die Jahre 2008-2013 (National Men's Health Policy 2008-2013, Ireland) [16]. Männergesundheit wurde in diesem Programm folgendermaßen definiert:

„Ein gesunder Mann besitzt ein Optimum an physischem, psychischem und sozialem Wohlbefinden und und nutzt seine Gesundheit für sein tägliches Leben" (4. Definition Männergesundheit).

Im Originaltext heißt es

Defining „men's health“: A healthy man is one, who is empowered to experience optimum physical, mental and social well-being and who experiences health as a resource for everyday living" [16; p.16].

\section{Definition Männergesundheit (Kanada)}

Die Men's Health Initiative of British Columbia, Canada, erstellte im Jahr 2010 eine Roadmap zur Männergesundheit, die Vorschläge zur Verbesserung der gesundheitlichen Lage und der Gesundheitsversorgung enthielt [16].

„Als Arbeitsdefinition für Männergesundheit gilt das Studium der Bedingungen oder Krankheiten, Risikofaktoren und Behandlungen, die spezifisch für Männer in deren Lebenslauf im Kontext ihres physischen, mentalen, emotionalen, sozialen und geistigen Wohlbefindens sind“ (5. Definition Männergesundheit).

Im Originaltext heißt es:

"The working definition of Men's Health is the study of conditions or diseases, risk factors and treatments, that are specific to males across their lifespan within the context of their physical, mental, emotional, social, and spiritual wellbeing" [16; p. 21].

\section{Definition Männergesundheit, Malaysia}

Der Asian Men's Health Report, der Ende 2013 im Rahmen der Malaysian Men's Health Initiative veröffentlicht wurde, definitert Männergesundheit folgendermaßen [12]: „Männergesundheit ist eine Disziplin, die physisches, psychisches und soziales Wohlbefinden von Männern in ihrem Lebenslauf (von der Kindheit zum Erwachsenenalter) 
fördert und sich mit Gesundheitsproblemen von Männern befasst. Ein multidisziplinärer Ansatz mit männerspezifischen Interventionen ist erforderlich, um die Lebensqualität und Lebenserwartung von Männern auf individuellem und Bevölkerungsniveau zu verbessern, und damit die Männergesundheit zu verbessern"

(6. Definition Männergesundheit).

Im Originaltext heißt es:

„Men's health is a discipline that promotes physical, mental and social well-being of men througout their life cylce (from boyhood to manhood) and addressing health problems related to men. A multi-disciplinary approach using male-specific intervention is required to improve the quality of life and life expectancy of men, both individually and as a population, which in turn improves men's health" [12].

\section{Definition Männergesundheit (USA)}

Die International Society of Men's Health (ISMH) und die Foundation of Men's Health, US, nahmen folgende Definition von Männergesundheit im Jahre 2013 an, die von Ridwan Shabsigh ausgearbeitet wurde [13]:

„Männergesundheit schließt ein:

(1) Männerspezifische Krankheiten und Bedingungen, bezogen auf die Anatomie wie Erkrankungen der Prostata, männliche sexuelle Dysfunktion, Hypogonadismus und Hodenerkrankungen bei Männern;

(2) Geschlechtsunabhängige Erkrankungen und Bedingungen mit höherer Prävalenz oder spezieller Bedeutung für Männer, wie kardiovaskuläre Krankheiten, das metabolische Syndrom, chronisch-obstruktuve Lungenkrankheiten, Lungenkrebs, Darm-, Blasen und Leberkrebs und Schizophrenie, Zwangsstörungen und Suizid;

(3) Verhaltensweisen, die häufiger bei Männern auftreten und Gesundheitsrisiken bedingen wie Rauchen, Substanzmissbrauch, Gewalt, geringe Inanspruchnahme von Gesundheitseinrichtungen und

(4) soziale Situationen, die die Mehrheit der Männer betreffen und negativen Einfluss auf ihre Gesundheit haben wie Dienst in der Armee, Kriege, Gefängnis, Baustellen, Bergbau und Schifffahrt" (7. Definition Männergesundheit).

Im Original heißt es:

"Men's health includes:

(1) Male gender-specific diseases and conditions related to the male anatomy, such as prostate diseases, male sexual dysfuntions, male hypogonadism and testicular and scrotal diseases;

(2) non gender-specific diseases and conditions with higher prevalence or special impact in men, such as cardiovascular disease; metabolic syndrome; chronic obstructive pulmonary disease; cancer of the lung, colon, bladder, and liver; and schizophrenia, obsessivecompulsive disorder, and suicide;

(3) behaviors that are more common in men and impose health risks, such as smoking, substancs abuse, violence, and low utilization on health services; and (4) social situations, involving a majority of men, that may have a negative impact on their 
health, such as the armed forces, wars, incarceration, construction, mining and shipping industries" [13].

\section{Bewertung}

Es liegen als Ergebnis einer umfassenden internationalen Literaturrecherche sieben Definitionen zum Begriff Männergesundheit vor, die im Zeitraum 1997 bis 2013 erstellt wurden.

Die erste Definition wurde im Jahre 1997 in Australien verfasst. Demzufolge ist Australien der Wegbereiter bei der Entwicklung der Definition Männergesundheit und Gesundheitsversorgung für Männer [4].

Die aufgeführten sieben Definitionen zur Männergesundheit entsprechen der Entwicklung von Männergesundheitspolitik vor allem in englischsprachigen Ländern (Australien, UK, Irland, Kanada, Malaysia (Asien) und USA).

Die Formulierung der Definition Männergesundheit erfolgte in diesem Zeitraum von einer durch männerspezifische Erkrankungen und Risikofaktoren geprägten Definition hin zu einer Definition, die zusätzlich die soziale Situation und Lebensbedingungen von Männern einbezog.

Die Definition des Men's Health Forum (UK) aus dem Jahre 2004 hat sich als sehr praktikabel erwiesen. Diese Definition wird in unverändertem Wortlaut dem Männergesundheitsbericht der Europäischen Union (2011) zu Grunde gelegt [11] und dient als Basis für die Definition von Männergesundheit, die dem weltweit ersten Programm einer nationalen Männergesundheitspolitik für die Jahre 2008-2013 für Irland zu Grunde liegt. Eine einvernehmliche globale Definition von Männergesundheit gibt es bisher nicht.

In drei Ländern werden Aspekte einer männerspezifischen Gesundheitsversorgung in die Definition einbezogen: in Australien (Definition 1), in Kanada (Definition 5) und in den USA (Definition 7). In England (Definition 2), USA, 2004 (Definition 3), Irland (Definition 4), Malaysia (Definition 6) wird dagegen der Versorgungsaspekt nicht als Bestandteil der Definition Männergesundheit erwähnt. In Irland wird die männerspezifische Gesundheitsversorgung als Bestandteil der Strategie und Männergesundheitspolitik betrachtet, nicht jedoch als Bestandteil der Definition. In Australien ist die männerspezifische Gesundheitsversorgung sowohl Bestandteil der Definition als auch der Männerpolitischen Landesstrategie.

\section{Definitionen zu Männergesundheit in deutschen Publikationen.}

Die deutschsprachige Literaturrecherche zu Definitionen "Männergesundheit" wurde in Datenbanken wie Google, Google Scholar, den DIMDI-Datenbanken, in „grauer Literatur“ und auf der Webseite von Wikipedia vorgenommen(s. Tab. 2).

\section{Deutsche Definition Männergesundheit}

Die im Jahr 2006 gegründete Stiftung Männergesundheit definierte Männergesundheit auf ihrer Webseite und publizierte sie im Männergesundheitsbericht 2013 [9; S. 7) Die Definition lautete: "Gesundheit bedeutet für jeden Mann etwas anderes: fit und jung, beweglich und schmerzfrei, körperlich wie seelisch nicht eingeschränkt für Beruf und Freizeit. Doch neben 
dem individuellen Empfinden bestimmt auch unser Umfeld, was gesund bedeutet: Ärzte und Krankenkassen, Medien und Meinungsmacher, Familie und Partner" [9] (1. Deutsche Definition Männergesundheit).

Diese Definition wurde Ende April 2013 auf der Webseite der Stiftung Männergesundheit durch die neu erstellte Definition abgelöst.

\section{Deutsche Definition Männergesundheit}

Im Lichtenberger (Berliner Stadtbezirk) Männergesundheitsbericht 2011 wird Männergesundheit folgendermaßen definiert [18]:

„Männergesundheit ist geprägt durch männlichen Lebensstil und Habitus, durch sozialstrukturelle Einflüsse auf die männliche Gesundheit und wird von gesellschaftlichen Bedingungen und Erwartungen beeinflusst. Männliches Verhalten und männliche Gesundheit werden auf der Individualebene (alltägliche Lebensführung) und der gesellschaftlichen Ebene (milieuspezifische Männergesundheit) entschieden" (2. Deutsche Definition Männergesundheit).

Der Lichtenberger Männergesundheitsbericht hat keine ISBN-Nummer, und erscheint deshalb nicht in Datenbanken. Er zählt zur "grauen Literatur“.

\section{Deutsche Definition Männergesundheit}

Die ursprüngliche Definition von „Praeventologische Beratung und Coaching” mit Sitz in Wuppertal, geführt von Gudrun J. Wiggershaus-Skriboleit, Wuppertal (www.praeventologie.eu) bis zum Jahre 2012 lautete [19]:

„Männergesundheit ist charakterisiert durch ein positives Gesundheitskonzept mit einem höheren Stellenwert von körperlichen Dimensionen und Leistungsfähigkeit, während das psychische Wohlbefinden einen geringeren Stellenwert einnimmt. Zu wichtigen personalen Ressourcen von Männern zählen ein hohes Selbstwertgefühl, Gefühl der körperlichen Stärke und Widerstandskraft sowie die Zuschreibung eigener Kompetenzen für die Bewältigung von psychischen und sozialen Belastungen. Familie oder Partnerbeziehung gelten als wichtige soziale Ressource."

(3. Deutsche Definition Männergesundheit) www.praeventologie.eu/gesundheit-s69 (Stand 26.9.2012)

\section{Deutsche Definition Männergesundheit}

Von 2012 bis 2013 wurde auf der Webseite der „Praeventologische Beratung und Coaching" folgende Definition publiziert [20\}, die inzwischen gelöscht wurde [21]: „Mit dem Begriff Männergesundheit werden diejenigen Aspekte von Gesundheit und Krankheit thematisiert, die für Männer und Jungen besonders relevant sind. Männer zeigen häufiger Verhaltensweisen und Lebensstile, die das Risiko für Verletzungen, Krankheiten, Tod erhöhen und sie sterben statistisch früher als Frauen".

(4. Deutsche Definition Männergesundheit)

(www.praeventologie.eu/gesundheit-s69 (Stand 11.4.2013) 


\section{Deutsche Definition Männergesundheit}

Auf der Webseite von Wikipedia gibt es folgende Definition von Männergesundheit, die nach dem Jahr 2007 erschien [22]:

„Unter Männergesundheit wird die körperliche, psychische und soziale Gesundheit von Jungen und Männern aller Lebensalter verstanden. Männergesundheit betrifft alle öffentlichen und privaten Bereiche wie Familie, Erziehung, Alltag, Kindergarten und Schule, Jugendarbeit, Politik oder das Arbeitsleben. Männergesundheit definiert sich und grenzt sich $a b$

1.) über spezifische körperliche Organe (Penis, Hoden, Prostata usw,

2.) über spezifische soziale und kulturelle Bedingungen des Aufwachsens und Lebensvollzugs von Jungen und Männern (Jungesein, Mannsein) sowie (teilweise daraus resultierend),

3.) über den genderbezogenen spezifischen Umgang mit Gesundheit (Bilder von Männlichkeit und Weiblichkeit)“

(5. Deutsche Definition Männergesundheit).

\section{Bewertung}

In Deutschland wurden bis zum Jahr 2014 drei Definitionen Männergesundheit in Männergesundheitsberichten und in der „grauen“ Literatur gefunden: die ursprüngliche Definition Männergesundheit der Stiftung Männergesundheit von 2006 bis 2013, die Definition des Lichtenberger Gesundheitsberichtes aus dem Jahre 2011 und die Definition auf der Webseite von Wikipedia. Zwei Definitionen von Gudrun J. Wiggershaus-Skriboleit wurden zwischenzeitlich von der Webseite der „Präventologie Coaching-Gesundheit" mit Sitz in Wuppertal entfernt, verbunden mit einer geänderten formalen und inhaltlichen Gestaltung der Webseite [19-21].

Die Literaturrecherche ergab, dass gegenwärtig drei der aufgeführten fünf Definitionen in Deutschland nicht genutzt werden. Demzufolge wird von den aufgeführten Definitionen nur die Definition Männergesundheit auf der Webseite von Wikipedia genutzt und die von der Stiftung Männergesundheit neu erstellte Definition aus dem Jahr 2013. Als Ergebnis der DIMDI-Recherche in deutschsprachigen Fachzeitschriften konnten keine Definitionen für Männergesundheit in Deutschland gefunden werden.

\section{Der Weg zur Definition durch die Stiftung Männergesundheit}

Die Formulierung der Definition erfolgte nach der durch Prof. Dr. Zinn modifizierten Delphi Methode, die bereits weiter oben beschrieben wurde.

In der ersten Runde legten die Experten folgende Mindestkriterien für die neu zu entwickelnde Definition fest:

a) Die WHO-Definition von "Gesundheit" soll die Grundlage für die Definition der Männergesundheit sein; 
b) Das salutogenetische Konzept von Antonovsky ist zu betonen;

c) Integration der Balance zwischen Risiko- und Schutzfaktoren;

d) Stärken und Schwächen von Männern in die Definition aufzunehmen;

e) Lebensfreude und persönliche Anerkennung zu berücksichtigen;

f) Integration des sozialen Hintergrundes und sozialer Unterstützung;

g) Spezielle Anforderungen an präventive Maßnahmen und die Gesundheitsversorgung aufzunehmen.

Die Themensammlung der zweiten Runde ergab folgende 15 Themengebiete:

1. Achtsamkeit gegenüber der eigenen Gesundheit

2. Gesunder Lebensstil

3. Akzeptanz der eigenen Schwächen

4. Eigene Gesundheitsaktivitäten

5. Sinn und Freude

6. Gesundheitlicher Fokus: Salutogenese

7. Soziale Klasse (Zugehörigkeit zu einer sozialen Klasse)

8. Akzeptanz wie ich als Mann bin

9. Krankheitsbezug

10. Einklang von Möglichkeiten und Zielen

11. Zugehörigkeit zu ethnischen Gruppen

12. Westeuropäischer Kontext

13. Gesellschaftliche Wertschätzung des Mannes

14. Männer - und Jungenspezifik

15. Internationaler Kontext

Durch die anschließende Gewichtung reduzierten sich diese auf acht Themengebiete:

1. Achtsamkeit gegenüber der eigenen Gesundheit ( 7 Punkte) 
2. Gesunder Lebensstil (7 Punkte)

3. Akzeptanz der eigenen Schwächen (6 Punkte)

4. Eigene Gesundheitsaktivitäten (5 Punkte)

5. Sinn und Freude (5 Punkte)

6. Gesundheitlicher Fokus: Salutogenese (5 Punkte)

7. Soziale Klasse (Zugehörigkeit zu einer sozialen Klasse) (5 Punkte)

8. Akzeptanz wie ich als Mann bin (4 Punkte)

Andere Begriffe wie „Einklang von Möglichkeiten und Zielen“, „Krankheitsbezug“, „Zugehörigkeit zu ethnischen Gruppen“ „Westeuropäischer Kontext“, „Gesellschaftliche Wertschätzung des Mannes“, „Männer- und Jungen-spezifik“, „Internationaler Kontext" erhielten nur 1 bis 2 Punkte und mussten deshalb nicht zwingend berücksichtigt werden.

In der dritten Runde wurde schrittweise die Definition Männergesundheit formuliert:

\section{Definition - Männergesundheit der Expertengruppe,}

(Berlin, 24. April 2013)

Männergesundheit umfasst diejenigen Dimensionen von Gesundheit und Krankheit, die insbesondere für Männer und Jungen relevant sind.

Gesundheit ist physisches, psychisches und soziales Wohlbefinden, das aus einer Balance von Risiko- und Schutzfaktoren entsteht, die sowohl in individueller, partnerschaftlicher als auch kollektiver Verantwortung liegen.

Als Schutzfaktoren wirken ein gesunder und achtsamer Lebensstil, Akzeptanz der eigenen Stärken, aber auch Schwächen als Mann, Sinnerfahrung und Lebensfreude, soziale Unterstützung und Anerkennung.

Die Risiko- und Schutzfaktoren sind besonders bei Männern in Abhängigkeit von Bildung, Herkunft, Einkommen und beruflicher Stellung ungleich verteilt.

Die gesundheitlichen Probleme der Männer bedürfen im gesamten Lebenslauf besonderer Präventions- und Versorgungsangebote, die größtenteils noch zu entwickeln sind.

[23, Webseite der Stiftung]

In der nächsten klassischen schriftlichen Runde erhielten alle Experten den Text und konnten noch finale Änderungen einbringen. Da alle dem Text so zustimmten, fand das Verfahren somit seinen erfolgreichen Abschluss. 


\section{Diskussion}

Begründung der inhaltlichen Aspekte des Definitionsbegriffes „Männergesundheit“

Das Bild von Männergesundheit war in den vergangenen 10 bis 20 Jahren überwiegend negativ geprägt. Geringere Lebenserwartung, höhere vorzeitige Sterblichkeit (0-64 Jahre), geringere Inanspruchnahme von Ärzten und Krankenhausleistungen, vor allem geringere Inanspruchnahme von Vorsorgeleistungen, zu wenig Aufmerksamkeit für den eigenen Körper und die eigene Gesundheit brachten Männern ein schlechtes Gesundheits-Image. Dieses negative Bild über Männergesundheit erwies sich als Nachteil für die Entwicklung von Gesundheitsstrategien [24]. In der Gesundheitsstrategie von Irland wird gesagt, dass es gegen männliche Verhaltensnormen verstoße, wenn diese ständig ihr eigenes Gesundheitsverhalten in Frage stellen müssten (It is against particular norms of masculine behaviour that men must constantly negotiate their own behaviour in relation to how they manage their health) [7]. Ein konstruktiver Weg sei gefragt [24].

Die WHO-Definition von Gesundheit geht nicht von negativen Einschätzungen der gesundheitlichen Lage aus, sondern von einer langfristigen Zielsetzung von Gesundheit als Zustand vollständigen körperlichen, psychischen und sozialen Wohlbefindens und nicht nur das Freisein von Beschwerden und Krankheit (WHO, 1946 [14]). Diese Zielsetzung gilt grundsätzlich für Frauen und Männer, jedoch ist offensichtlich, dass der Gesundheitsstatus und die Gesundheitsbedarfe von Männern und Frauen teilweise unterschiedlich sind. Deshalb ist es notwendig, die Gender-Unterschiede auch in spezifischen Definitionen für Frauen- und Männergesundheit konzeptionell zu berücksichtigen.

Die internationale Definitionsfindung zeigt, dass die erste Definition von Männergesundheit vor fast 20 Jahren von der Definition „Women's Health” abgeleitet wurde. In den vergangenen 10 Jahren wurde in mehreren Ländern versucht, spezifische Definitionen von Männergesundheit zu formulieren. Je stärker die Bewegung um „Männergesundheit“ weltweit wird, desto wichtiger wird eine Verständigung um eine international akzeptierte Definition von Männergesundheit [31]. Die WHO-Definition von Gesundheit und die Definition des Men's Health Forum (England), die dem EU-Gesundheitsbericht „State of Men's Health in Europe" zu Grunde liegen [11;3], treffen schon viele Aspekte eines salutogenetischen und männerspezifischen Herangehens.

In der von der deutschen Expertengruppe erarbeiteten Definition wurde bewusst der Hinweis auf spezifische Krankheiten oder Verhaltensweisen vermieden, die Defizite der Männergesundheit und ihrer Verhaltensweisen in den Vordergrund stellen, z.B. der "risikoaffine Mann“; der „Arztverweigerer" oder der „Vorsorgemuffel“ [25]. Das entspricht der internationalen Literatur. Die WHO-Definition von Gesundheit wurde bei der Definitionsfindung zu Grunde gelegt und wissenschaftliche Literatur aus Deutschland herangezogen.

Als gute Grundlage einer Definitionsfindung diente die Definition von „Gesundheit“ von Klaus Hurrelmann [26] aus dem Jahre 2010, „Gesundheit als „Zustand des objektiven und subjektiven Befindens einer Person zu betrachten, der gegeben ist, wenn diese Person sich in den physischen, psychischen und sozialen Bereichen ihrer Entwicklung im Einklang mit 
den eigenen Möglichkeiten und Zielvorstellungen und den jeweils gegebenen äußeren Lebensbedingungen befindet." In seinem Verständnis, das die Brücke zu den interdisziplinär orientierten Gesundheitswissenschaften schlägt, ist Gesundheit ein angenehmes und durchaus nicht selbstverständliches Gleichgewichtsstadium von Risiko- und Schutzfaktoren, das zu jedem lebensgeschichtlichen Zeitpunkt immer erneut in Frage gestellt ist. Gelingt das Gleichgewicht, dann kann dem Leben Sinn und Freude abgewonnen werden, es ist eine produktive Entfaltung der eigenen Kompetenzen und Leistungspotentiale möglich, und es steigt die Bereitschaft, sich gesellschaftlich zu integrieren und zu engagieren [26].

Die Deutsche Gesellschaft für Mann und Gesundheit grenzte Männergesundheit folgendermaßen ein (2010) [27]:

„Männergesundheit ist ein individuelles und ein gesellschaftliches Thema. Demnach geht es darum, den einzelnen Mann anzusprechen, ihm die Verantwortung für die eigene Gesundheit nahe zu bringen und ihn bei deren Wahrnehmung zu stärken. Doch es geht ebenso um die Lebensbedingungen der Männer in unserer Gesellschaft. Diese gilt es zu beleuchten, Probleme aufzudecken und für deren Lösung einzutreten. Nur ein ausgewogenes Verhältnis zwischen der Ansprache des Einzelnen und der Thematisierung gesellschaftlicher Bedingungen ist der Sache angemessen und bringt die Männergesundheitsdiskussion voran" [27].

Das Themenfeld Männergesundheit hat sich in Deutschland nur mühsam und sehr punktuell entwickelt. Schwerpunkte waren bis jetzt „Männerkrankheiten“ und Probleme bei der "Sexualität". Deshalb war es besonders wichtig, Männergesundheit umfassender zu definieren, um ein öffentlich akzeptiertes Verständnis von Männergesundheit zu entwickeln. Nur eine umfassende Definition von Männergesundheit kann dazu beitragen, eine angemessene Betrachtung in der Öffentlichkeit und in der Gesundheitspolitik zu etablieren.

Der Bezug der neuen Definition von Männergesundheit zur WHO-Definition von Gesundheit ist im zweiten Absatz dargestellt. Der Vorschlag schließt aber zusätzlich die Balance von Risiko- und Schutzfaktoren des Individuums ein. Der Gesundheitswissenschaftler K. Hurrelmann hat zutreffend bei der Definition von „Gesundheit" [26] auf das notwendige Gleichgewicht von Risiko- und Schutzfaktoren hingewiesen, auf das wir hier Bezug genommen haben.

Weiterhin wird die kollektive Verantwortung betont. Dadurch wird einer Tendenz zu einer ausschließlich auf das Individuum zielenden Thematisierung von Gesundheitsverantwortung entgegengetreten und die Bedeutung von Familie, Betrieb und intermediären Institutionen herausgestellt. Das entspricht der Definition von Männergesundheit durch das Men's Health Forum England, in dem Verbesserungen von Männergesundheit auf individuellem und bevölkerungsbezogenem Niveau thematisiert werden [3]. Außerdem wird Konsens mit der neu angestoßenen Diskussion der WHO im European Health Report 2012 zur Bedeutung von Well-being im New Public Health Kontext, d.h. der staatlichen Verantwortung für Gesundheit und Wohlergehen, signalisiert [28].

Die „alte“ Definition „Männergesundheit“ der Stiftung Männergesundheit [29] besagte, 
dass Männer über „Männergesundheit“ ganz unterschiedliche Vorstellungen haben.

Der Gedanke der individuellen Wünsche und Vorstellungen, der bereits in der bisherigen Definition „Männergesundheit" enthalten war, sollte nicht nur deshalb Eingang in die neue Definition finden. Vielmehr entspricht das auch der Individualisierung der Lebensläufe sowie anderen Tendenzen postmoderner Gesellschaften, auf die sich auch die Gesundheitspolitik einstellen muss.

Die bis zum Jahr 2011 veröffentlichte Definition zu Männergesundheit von WiggershausSkriboleit stimmte nicht mit anderen Definitionen zu Männergesundheit in Deutschland überein [19], da sie in einer normativen Weise ein positives Bild von Männergesundheit vermittelte. Die Definition wurde durch eine zweite Definition bis zum Jahr 2014 ersetzt [20], ist jedoch seit kurzem von der Webseite entfernt worden [21].

Ein Abgleich mit einer deutschen Definition von Frauengesundheit erfolgte nicht, da im Bericht zur gesundheitlichen Situation von Frauen in Deutschland [30] des Bundesministeriums für Familie, Senioren, Frauen und Jugend aus dem Jahre 2001 zwar Frauen- und Männergesundheit voneinander abgegrenzt werden, aber keine Definition von Frauengesundheit enthalten ist.

Die Deutsche Gesellschaft für Mann und Gesundheit e.V. [27] betonte den Einfluss der sozialen Bedingungen auf die Gesundheit des Individuums, die in der Definition berücksichtigt wurden.

Mit dieser Definition liegt eine erweiterte Definition von Männergesundheit vor, die individuelle, partnerschaftliche, kollektive Verantwortung und die bedeutung der Gesundheitsversorgung für Männergesundheit einbezieht.

Sie kann als Basis für weitere Forschungsarbeiten und konkrete Maßnahmen zu diesem Thema dienen. Eine umfassende Definition von Männergesundheit sollte die verschiedenen Konzepte von Männlichkeit und Diversität von Männlichkeiten mit den Dimensionen Alter, Klasse, ethnische Herkunft, sexuelle Identität, Bildung, Weltanschauung / Religion mit einbeziehen. Diese Aspekte wurden von der Expertengruppe im Themenkatalog für eine Definition von Männergesundheit genannt, im Auswahlverfahren der Kriterien vorerst zurückgestellt. Aber diese Kriterien sollten als Basis für weitere Forschungsarbeiten zum Thema Männergesundheit dienen.

\section{Merksätze}

Mit dieser Publikation wollen wir dazu beitragen:

- Die Diskussion über Männergesundheit weltweit und speziell in Deutschland voranzubringen,

- Eine Definition von Männergesundheit zu finden, die dem aktuellen Erkenntnisstand und den spezifischen Bedürfnissen der Männer entspricht. 
für Aktionspläne zur Verbesserung der gesundheitlichen Lage von Männern, vor allem zur Reduzierung vorzeitiger Sterblichkeit und Invalidität und Erhöhung ihrer Lebenserwartung und schließt Prävention, medizinische Versorgung, Beeinflussung des Verhaltens und soziale Differenzierung ein.

\section{Fazit für die Praxis}

Die Public Health Relevanz der vorgestellten Definition „Männergesundheit“ besteht darin, den salutogenetischen Ansatz über Gesundheitspotentiale von Männern in den Mittelpunkt einer Definition zu stellen, um auf dieser Grundlage „Aktionspläne für Männergesundheit“ zur Verbesserung der gesundheitlichen Lage von Männern zu initiieren.

Die Definition "Männergesundheit" ist auf der Webseite der Stiftung Männergesundheit publiziert; sie ist die Grundlage für die Fortsetzung der wissenschaftlichen Diskussion. 


\section{Literatur}

1. White A. Men's health: The challenges ahead. Editorial jmhg 2004, Vol 1, No. 4: 296-299

2. Fletcher R. Report on men's health services. Prepared for NSW Department of Health by Men's Health Advisory Group. Family Action Centre, University of Newcastle. NSW 2318, Australia, 1997

3. Men's Health Forum (MHF). MHF action briefing. Men's health. Getting it sorted: a policy programme for men's health. London: Men's Health Forum; 2005.

www.menshealthforum.org.uk/../gettingsorted2004.pdf; Stand 12.01.2015

4. Porche DJ. A call for American Journal of Men's Health. AJMH 2007. Editorial. 2007;1(1):5-7

5. Porche DJ, Willis DG. Nursing and men's health movement: Considerations for the $21^{\text {st }}$ century. Nurs Clin N Am 2004; 39: 251-258

6. Smith JA. Addressing men's health policy concern in Australia: what can be done?. Australia and New Zealand Health Policy 2007; 4:20. Doi: 10.1186/1743-8462-4-20

7. Richardson N, Smith JA. National men's health policies in Ireland and Australia: What are the challenges associated with transitioning from development to implementation. Public health 2011; 125: 424-432

8. Stiftung Männergesundheit Berlin (Hrsg.): Erster Deutscher Männergesundheitsbericht 2010. Ein Pilotbericht. Doris Bardehle, Matthias Stiehler. W.Zuckschwerdt Verlag München, 2010

9. Stiftung Männergesundheit Berlin (Hrsg.): Männergesundheitsbericht 2013. Im Fokus: Psychische Gesundheit. Verlag Hans Huber, 2013

10. Robert Koch-Institut (Hrsg): Gesundheitliche Lage der Männer in Deutschland 2014. Beiträge zur Gesundheitsberichterstattung des Bundes. RKI, Berlin, 2014

11. European Union. Directorate-General for Health \& Consumers. The State of Men's Health in Europe. Extended Report of the European Union. Definition of Men's Health p. 25. Brussels, 2011 www.ec.europa.eu/health/population_groups/.../men_health_extended.en.pdf. Stand 14.01.2015, p. 25) 
12. Malaysian Men's Health Initiative (Hrsg): Asian Men's Health Report 2013. Published by Malaysian society of andrology and the study of ageing male, Selangor, Malaysia, 2013

13. Shabsigh R. A new multidisciplinary approach to mens's health. Editorial. Journal of Men's Health 2013; vol 10, No. 1: 1-2. DOI: 10.1089/jomh.2013.1500

14. Weltgesundheitsorganisation (WHO). Preamble to the Constitution of the World Health Organization as adopted by the International Health Conference, New York, 19-22 June, 1946; signed on 22 July 1946 by the representatives of 61 States (Official Records of the World Health Organization, no. 2, p. 100) and entered into force on 7 April 1948. 2http://www.who.int/healthsystems/hss-glossary/en/index5.html; Stand: 26.02.2015

15. Bortz DJ, Döring N. Forschungsmethoden und Evaluation. Springer Verlag,2006: $261 \mathrm{ff}$ )

16. Minister for Health and Children: National Men's Health Policy 2008-2013, Ireland, Main editor: Richardson N. Published by the Stationary Office, Dublin 2008

17. Mens Health Initiative of British Columbia, Canada. A Roadmap to Men's Health. ( $)$ D. Bilsker, L. Goldenberg, J.\& Davison J, 2010: page 21

18. Bezirksamt Lichtenberg von Berlin, Hrsg. Man(n), wie geht's? Lichtenberger Männergesundheitsbericht 2011. Berlin: Techniker Krankenkasse; 2011: 192 S. www.berlin.de/ba-lichtenberg/buergerservice/gesundheit; Stand: 19.09.2013

19. Wiggershaus-Skriboleit G J. Präventologische Beratung und Coaching mit Sitz in Wuppertal. Definition von Männergesundheit. http:www.praeventologie.eu, Stand: 26. 09. 2012

20. Wiggershaus-Skriboleit G J. Präventologische Beratung und Coaching mit Sitz in Wuppertal. Definition von Männergesundheit. http:www.praeventologie.eu, Stand: 11.04.2013

21. Wiggershaus-Skriboleit G J: Präventologie Coaching Gesundheit mit Sitz in Wuppertal. www.coaching-gesundheit.de, Stand 21.01.2015

22. Wikipedia: Definition Männergesundheit.

http://de.wikipedia.org/wiki/Männergesundheit. Stand 16.01.2015

23. Stiftung Männergesundheit Berlin. (Gobelin)-Definition Männergesundheit 24. April 2013 in deutscher und englischer Sprache. In: Men's Health Report 2013. In Focus: Mental health. Condensed version. Hrsg. Stiftung Männergesundheit. Okt. 2013. www.stiftung-maennergesundheit/dieStiftung/Links/Definition (Stand 21.01.2015)

24. White $A$, McKee $M$, Richardson $N$ et al. Europe's men need their own health strategy. BMJ 2011; 343:d7397 doi: 10.1136/bmj.d7397. Published 29. Nov.2011 
25. Meuser M: Der kranke Mann. In: Dinges M (Hrsg.): Männlichkeit und Gesundheit im historischen Wandel (1850 - 2000). Franz Steiner Verlag, Stuttgart: 2007: 73-86

26. Hurrelmann K. Definition von Gesundheit. In: Hurrelmann D: Gesundheitssoziologie, Weinheim: Juventa; 2010

27. Stiehler M. Vorwort der Gesellschaft für Mann und Gesundheit e.V.. In: Bardehle D, Stiehler M. Erster Deutscher Männergesundheitsbericht 2010. München: W. Zuckschwerdt; 2010, S. VII

28. World Health Organization. Regional Office for Europe. Der Europäische Gesundheitsbericht 2012. Ein Wegweiser zu mehr Wohlbefinden. World Health Organization 2013. www.euro.who.int/_data/.../The-European-Health-Report_2012charting-the-way-to-well-being; Stand 26.02.2015

29. Stiftung Männergesundheit Berlin. Alte Definition Männergesundheit: 20 April 2013. www.stiftung-maennergesundheit/Definition; Stand: 20.04.2013

Publiziert in: Weißbach L, Stiehler M. Männergesundheitsbericht 2013. Im Fokus:Psychische Gesundheit. Verlag Hans Huber,2013, S. 7

30. Bundesministerium für Familie, Senioren, Frauen und Jugend (Hrsg): Bericht zur gesundheitlichen Situation von Frauen in Deutschland 2001. Schriftenreihe Band 209, Verlag Kohlhammer GmbH Stuttgart,2001

31. European Men's Health Forum (EMHF). Editors: Wilkins D, Savoye E: A review of policy and progress across 11 countries. Published by the EMHF. Spring 2009 